https://doi.org/10.52449/1857-4114.2021.37-1.01

CZU: 796.032.2:339.1

\title{
SPORTS SERVICES, PRODUCT OF PHYSICAL CULTURE ACTIVITIES
}

\author{
Budevici-Puiu Liliana ${ }^{1}$, ORCID: 0000-0001-7170-2016 \\ ${ }^{1}$ State University of Physical Education and Sport, Chisinau, Republic of Moldova
}

\begin{abstract}
Currently, the sports industry is generated by a professional movement. The association policy has strong repercussions on the degree of professionalism, efficiency and freedom of initiative in the activity of professionals in the field. In modern society, professional sports are presented as a type of business, as an important part of the entertainment industry, as well as one of the most complex forms of trade. In other words, we can mention that professional sport is a type of entrepreneurial activity, whose purpose is to make a profit from the sale of the competitive show. In the last decade, professional sports have actively developed, both nationally and internationally, so that the practice of entering into partnerships has expanded, if we refer to athletes who are interested in working in foreign clubs. The professionalization of sport is an objective, inevitable process that contributes to an increase in the effectiveness, technicality, aesthetics and entertainment of sport. In addition, placing sport on a new organizational and economic basis gives the state the opportunity to supplement its budget with additional financial resources [3, 4]. However, the characterization of the dynamics of the development of professional sports, reflected by several authors, attests to the fact that, through its professionalization, sport loses its function and humanistic role in society. "Professional sport is a social disaster, because its product does not only consist of victories and results that constitute the glory and image of a state, but involves people who have lost their health" [5]. In professional sports, the principle of the Olympic sport "Fair - play" loses its meaning, leaving room for the one referring to obtaining "Victory at any cost" (intimidation of the opponent, aggression, fraud, severe psychological pressure on the competitor out of competition and in the competitive process). We are currently witnessing the commercialization of sport and the fact that the Olympic movement is beginning to be a "synthesis of sports performance, advertising technology and public policy". Olympic sports are distancing themselves from mass (ordinary) sports, arguments highlighted by the following aspects: the rapid growth of sports achievements is not in line with those of the physical condition of the population. At the same time, Olympic sports guide and ensure the subsidization of huge sums from a state budget. For example, the budget for the Tokyo Olympics was 1.6 - 1.8 trillion Japanese yen, or about 14.5 - 16.2 billion euros for 33 sporting events, 47 disciplines and 324 events, at which was attended by over 12,000 athletes [7].
\end{abstract}

Keywords: sports, professionalization, marketing, Olympism, Olympic movement.

Introduction. The sports events entered the Olympic program with their own rules and traditions, with a universal structure and a decisive role of the economic component, with an advanced management, with balanced rights and responsibilities of qualified athletes, as well as with organizational and management structures based on democracy principles [3, 4]. Olympic sport has developed and become a multi-billion dollar business, covering all corners of the globe [6]. Throughout modern history, sport has been based on the philosophy of Olympism, on the fundamental principles of the Olympic 
movement. According to the Olympic Charter, Olympism is "a mixture of sport, culture and education" or "a lifestyle based on joy, built on effort, the educational value of good example and respect, universal fundamental ethical principles" [6]. However, Olympism is also characterized by anti-human phenomena, such as chauvinism, referee bias, subjectivity and hostility of fans, and last but not least by the disorders brought to the health of athletes as a result of intensive training processes. Doping has become an almost indispensable attribute of the modern Olympic movement, and manipulation and corruption can be easily identified in large-scale sports activities. Thus, the falsification of sports results leads to the destruction of athletes' health and contributes to the fact that the performance obtained is determined by the achievements of chemistry and pharmacology, and not by the athlete himself. However, Olympism is based primarily on universal human values such as [1]:

- physical and spiritual improvement of those who practice it;

- a human and active life;

- an effective spirit of rivalry;

- special respect and an inherent peacekeeping function.

The subject approached in the research is a very wide one, as the Olympic sports events are in a continuous change, which is led by sports managers, referees, players, fans, as well as the generation of massive income from sports. The emergence of new sports (freestyle, short track, bodybuilding, sports aerobics, synchronized swimming, hang gliding, sports windsurfing, etc.) attracts new competitive events in this field. Moreover, the formation of sports has become possible due to the modernization of the existing typology of sports events, the interaction of sports with other branches of culture (sports dancing, rhythmic gymnastics), the influence and mutual enrichment of various sports, scientific and technological advances.
The purpose of the research is to analyze trends in the development of Olympic sports in the context of marketing and professionalization.

Research methods used in research have materialized in the general theories (analysis, synthesis, abstraction and generalization) and methods of scientific knowledge (study of literature analysis and description).

Professionalization marks the transition from amateurism to professionalism, and in order to better understand this aspect, we will further present some essential arguments in this regard. Professionalization is a process that, according to Richard Wittorski [5], is situated on two essential levels:

- professionalization of actors: it refers to the training of individuals for one of the already existing professions in the field of reference;

- professionalization of activities: transformation of an activity into autonomous profession.

The author attributes to the concept of professionalization three meanings:

- "Professionalization - profession" - the establishment of an autonomous social group;

- Assistance in work flexibility "Professionalization - Work efficiency"

- The process of training the professional through training - "Professionalization Training"

In the world of sports the words "occupation" and "profession" are used, synonymous concepts.

The coexistence of several statuses (management, executive and subordinate staff, technical-tactical, medical, kinetotherapy training, etc.), whose boundaries often remain unclear, creates internal tensions and important power games to occupy a central place in the functioning of sports organizations.

The content of the concept of "professional sport" refers to certain groups of professional athletes $[3,4]$ :

1) athletes who successfully participate in both the Olympic Games and the world 
championships, as well as a series of cup starts and commercial ones;

2) athletes who have high results, but who do not meet the requirements to successfully participate in major competitions;

3) veteran athletes, specialized especially in sports games, such as martial arts, figure skating.

The professionalization regime and all the obligations deriving from it (sports training, technical-tactical training, level of competitions) determined the sports coaches / managers to considerably develop the training process. Also, the big TV channels started to pay a lot to broadcast large-scale competitions, and the professional athletes proceeded to conclude substantially paid contracts. Therefore, more and more clubs started to organize their sports life with their professional teams, the technical and medical staff registering at the same time, a considerable increase. Players were also given the opportunity to change their personal lives in order to withstand the new workloads. Thus, they were forced to take certain periods of recovery and change their diet, often leading to some abuse, fatigue and, therefore, incompatibility with the weekly work regime and the performance they had to achieve in a given competition [2].

Currently, we are witnessing a meticulous and precise management of the activity of the performance athlete. The most advanced means are implemented, and the athlete is now monitored, controlled, cared for and managed in the smallest detail. The medical staff uses the most advanced medical instruments, applying maintenance and recovery procedures to the athletes at the highest level. The professional player has become a true athlete, capable of high performance. All this, obviously, favored the immediate increase of the level and time of play. Indeed, we can see that the actual playing time in a match (about eighty minutes, minus the downtime due to refereeing and injury) has increased considerably. This increase in playing time is also the result of better training (especially physical training) of professional athletes able to run, jump, push, tackle techniques and tactics much better than before. Therefore, this strengthens the quality of the show offered during the competitions and generates the envy of broadcasters, advertisers, viewers and fans" $[1,2]$.

Another unresolved issue in professional sports is the sports calendar, as the number of competitions has increased, which affects the training and recovery of professional athletes in a timely manner to participate in new sporting events. It is therefore imperative that athletes rest and prepare to avoid an excessively high rate of injuries (accidents at work) and unavoidable drug abuse, which often go hand in hand with money from wellpaid contracts, and with the overloads of professional athletes. A feature of the modern competition system is not only the stability of the competition calendar, but also the attachment to sports facilities (venue). Thus, the organization and development of a major sporting event is a complex task, which involves not only the construction of sports facilities, but also the formation of an entire infrastructure [3]. The use of modern management in the organization and conduct of competitive tournaments for a long time has contributed not only to the popularization of sport as a whole, to the development of Olympic events, but has made possible the transformation of such tournaments into major commercial events, revenues of national federations and public authorities. The professionalization of sport requires that the problems of social protection of athletes, the mechanisms for regulating labor relations and the problems they face in society be taken into account. It is also important the influence of specialists of different profiles on the training of athletes and their participation in competitions. The training and coaching centers have a special impact on athletes, as strong stimuli for the development of Olympic sports and for creating a competitive 
environment, generating changes in the value orientations of athletes [4].

The separate development of Olympic and professional sports has contributed to the formation of distinct organizational systems, competition systems and different training methods. However, in the new socio-economic conditions, the trends of professionalization and commercialization of Olympic sports have clearly manifested themselves. The principles of amateur sport, in its traditional understanding, on which the functioning of Olympic sport was based, came into conflict with the realities of their modern forms of development, which were the basis for qualitative organizational and legal changes [3]. The interest for the Olympic competitions was determined by the high and expected quality of the competitive activity of the participants, by the intrigue and the unpredictability of the final result. To create a fierce competition, the organizers began to invite a limited number of athletes (the most popular), leaders of the season, to participate in competitions and demonstrate high sports results.

Forcing sports training sometimes limits the ability to perform complex (professional) activities efficiently and reliably, reduces motivation, undermines the aspiration system, orients the athlete's values due to injuries, stops increasing results, often leading to the end of a sports career. The situation is aggravated by the specific feature of professional sports that is clearly manifested in Olympic sports - creating and maintaining an atmosphere of fierce competition, without which the sport loses its appeal to viewers, the interest of television and sponsors.

Today, the world is facing the acute problem of increasing the duration of a sports career. However, as sports practice shows, most athletes, after completing their sports careers, will face the problem of health, social adjustment, which will naturally affect the quality of life.
We can also mention that the professionalization and marketing of modern sports has become the reason for the formation of a social protection system for outstanding athletes. Thus, an important role in protecting the interests of athletes is attributed to intermediary agencies, which conclude commercial contracts on their behalf, investing the money earned in a particular business, which largely determines their future existence after completing their sports career, a faster adaptation to new living conditions. However, compared to professional sport, the social protection system in Olympic sports is still in its infancy. Unfortunately, today many athletes, especially in the countries that appeared in the post-Soviet space, face the problem of social protection - insufficient wages, minimum material guarantees in the form of pensions / annuities, a sharp deterioration in working conditions, lack high quality technical and material support, lack of developed training infrastructure (stadiums, arenas, sports equipment and facilities), lack of specific regulations to create the conditions and take measures to ensure adequate protection of health and safety of participation in competitions, the departure of highly qualified specialists to work abroad on higher salaries. An important way of forming a social protection system in different countries is the payment of personal scholarships to high-class athletes, personal pensions for athletes who have ceased their sports activities, all of which are regulated by law. However, as evidenced by the practice of sport in recent years, many gifted athletes are forced to look for better conditions for training in other countries $[1,3]$.

The influence of professionalization and marketing trends in Olympic sports, occurred against the background of important changes that affected its further development:

$\checkmark$ adoption of a regulatory framework governing payments and revenues allocated to athletes, the activity of their representatives, advertising agencies, broadcasting international competitions, etc. .); 
transforming the competition system, integrating the formats of Olympic and professional sports into a single competition system and developing prize / grant funds for the organization of large-scale tournaments;

$\checkmark$ training sports elites among the stars of world sports, whose lifestyles, hobbies and incomes are constantly in the visual field of television and the press;

$\checkmark$ formation of a social protection system for performance athletes.

In conclusion we can mention that:

$\checkmark$ professionalization and marketing of sports, of the modern system of competitions affects the training of athletes;

$\checkmark$ in sports, a large number of qualified specialists have been trained in certain specializations - coaches, sports doctors, kinetotherapists, masseurs and for whom sport has become a sphere of professional activity;

$\checkmark$ a coach has a significant influence on the training of an athlete, on whose professionalism depends his career in perspective (sports practice abounds in examples with reference to the high level of qualifications and organizational and pedagogical skills of coaches that can inspire athletes to achieve results remarkable, but also examples that represent a danger to the successful career of an athlete);

$\checkmark$ In the context of the commercialization of Olympic sports, the system of coach-sports relationship has changed (athletes began to earn more money than their mentors, thus becoming more independent, being guided not by methodical considerations but by economic ones when making decisions to and easily exchange coaches with more "comfortable" ones);

$\checkmark$ the mistakes of qualified coaching staff and their uncoordinated activities can lead to serious consequences that give rise to trauma, illness and, consequently, lead to the end of a sporting career;

$\checkmark$ it is necessary to continuously improve the training of highly qualified athletes, taking into account professional and marketing trends, to identify effective approaches with a focus on solving the various strategic objectives (achieving the highest results at the Olympic Games, in major tournaments, prestigious, there must be a rational combination of performance and success);

$\checkmark$ in modern socio-economic conditions, there is a process of integration into a single whole of two types of sports with the highest achievements - Olympic and professional. Thus, performance sports acquire the characteristics of a mixed form of organization, which combines various priorities and criteria - obtaining the highest results at the Olympic Games, the number of medals won, the team's place in the team's unofficial competition and business.

\section{References:}

1. Kozlova E.K., Rabin M.F. (2016). The dynamics of the effectiveness of the competitive activity of the most powerful athletes in the world during the Olympic year. [in Russian] In: Science in Olymp. Sports, no. 4, p. 23-34.

2. Imas E.V., Borisova O.V. (2017). Professional tennis: problems and development perspectives: [monograph] [in Russian]. Kiev: Publishing House “Olymp. LIT”, 2017. 288 p.

3. Platonov V.N. (2015). The system of training athletes in Olympic sports. General theory and its practical applications: manual [for trainers] [in Russian]. Kiev: Olimp. lit., 2015. 680 p.

4. Platonov V.N., Bubka S.N, Bulatova M.M. and others. (2009). Olympic sports. [in Russian] Kiev: Olymp. lit. T.2. 696 p.

5. Wittorski R. (2008). Science, International Journal of Educational Research and Training for Adults, "Professionalization", Summary Note, p. 15.

6. White Paper on Sport

7. www. diez.md 